\title{
The Boltzmann Constant: Evaluation of Measurement Relative Uncertainty Using the Information Approach
}

\author{
Boris Menin \\ Refrigeration Consultancy Ltd., Beer-Sheba, Israel \\ Email: meninbm@gmail.com
}

How to cite this paper: Menin, B. (2019) The Boltzmann Constant: Evaluation of Measurement Relative Uncertainty Using the Information Approach. Journal of Applied Mathematics and Physics, 7, 486-504. https://doi.org/10.4236/jamp.2019.73035

Received: February 10, 2019

Accepted: March 9, 2019

Published: March 12, 2019

Copyright (อ 2019 by author(s) and Scientific Research Publishing Inc. This work is licensed under the Creative Commons Attribution International License (CC BY 4.0).

http://creativecommons.org/licenses/by/4.0/

\section{c) (i) Open Access}

\begin{abstract}
The purpose of this work is to prove that only by applying a theoretically sound information approach to developing a model for measuring the Boltzmann constant, one can justify and calculate the value of the required relative uncertainty. A dimensionless parameter (comparative uncertainty) was proposed as a universal metric for comparing experimental measurements of Boltzmann constant and simulated data. Examples are given of applying the proposed original method for calculating the relative uncertainty in measuring the Boltzmann constant using an acoustic gas thermometer, dielectric constant gas thermometer, Johnson noise thermometer, Doppler broadening thermometer. The proposed approach is theoretically justified and devoid of the shortcomings inherent in the CODATA concept: a statistically significant trend, a cumulative value of consensus or a statistical control. We tried to show how a mathematical-expert formalism can be replaced by a simple, theoretically grounded postulate on the use of information theory in measurements.
\end{abstract}

\section{Keywords}

Boltzmann Constant, Comparative Uncertainty, Information-Based Approach, Relative Uncertainty

\section{Introduction}

In 2018, for the first time in the history of modern science, a unique decision was taken: a modification of the International System of Units (SI). It is based on the redefinition of the four basic units of kilogram, ampere, kelvin and mole of the SI in terms of fundamental physical constants. The basic unit of thermodynamic temperature $\theta$, kelvin, was based on a fixed numerical value of the 
Boltzmann constant $k=1.380649 \times 10^{-23} \mathrm{~J} \cdot \mathrm{K}^{-1}$. This became possible only due to the unique methods of measuring the Boltzmann constant. However, the numerical value of the Boltzmann constant is fixed only when special requirements are met for the relative uncertainty of measurement [1]: the relative standard uncertainty of the adjusted (mean) value of $k$ is less than $1 \times 10^{-6}$; the determination of $k$ is based on at least two fundamentally different methods, of which at least one result for each shall have a relative standard uncertainty less than $3 \times$ $10^{-6}$. To calculate the value of relative uncertainty, modern statistical methods and supercomputers are used. Besides, at each stage of evaluating the results, an expert analysis of the obtained data is carried out. It means to use the specialist's own intuition, knowledge and experience (one's personal philosophical leanings [2]). Such a situation does not exclude the presence of a biased statistical expert, motivated by personal convictions or preferences. It should be mentioned that the relative uncertainty method for identifying the measurement accuracy does not indicate the direction in which one can find the true value of a fundamental physical constant. In addition, it involves an element of subjective judgement [3].

In contradiction with CODATA (The Committee on Data for Science and Technology) statistics-expert techniques, the proposed method [4] [5] is a theoretically grounded approach, based on the theory of information. The approach begins with a simple concept, for example each model of the phenomenon includes a certain amount of information. It is assumed that the end result will be one value that predicts the model uncertainty prior to the experiment or computer simulation. The information-based approach serves as a practical tool for checking the lowest achievable model uncertainty. It also creates the right scientific discussion about the maximum optimal number of variables recorded.

In this paper, we have focused on a very important application of the information-based method: analysis of the relative uncertainty in the measurements of the Boltzmann constant.

\section{Information Measure Approach}

The approach, called the $\mu_{\mathrm{SI}}$-hypothesis, was formulated in [6]. It focused on calculating the lowest comparative uncertainty of the researched quantity and based on principles of information and similarity theories with the usage of the International System of Units (SI). Following it, a certain uncertainty exists before starting experiment due only to the known recorded number of quantities. In turn, the dimensionless comparative uncertainty $\varepsilon$ of the dimensionless quantity $u$, which varies in a predetermined dimensionless interval $S$, for a given number of selected physical dimensional quantities $\boldsymbol{z}^{\prime \prime}$, and $\boldsymbol{\beta}^{\prime}$ (the number of the recorded base quantities) can be determined from the relation:

$$
\varepsilon=\Delta u / S \leq\left[\left(z^{\prime}-\beta^{\prime}\right) /(\Psi-\xi)+\left(z^{\prime \prime}-\beta^{\prime \prime}\right) /\left(z^{\prime}-\beta^{\prime}\right)\right]
$$

where $\Delta u$ is the dimensionless uncertainty of the physical-mathematical model describing the measurement experiment: 
(1) $\xi$ is the number of base quantities with independent dimension; SI includes the following seven ( $\xi=7)$ base quantities: $L$ is the length, $M$ is mass, $T$ is time, $I$ is electric current, $\Theta$ is thermodynamic temperature, $J$ is force of light, $F$ is a number of substances. The dimension of any derived quantity $q$ can only express a unique combination of dimensions of base quantities in different degrees [7]:

$$
q \supset L^{l} \cdot M^{m} \cdot T^{t} \cdot I^{i} \cdot \Theta^{\Theta} \cdot J^{j} \cdot F^{f} .
$$

(2) $l, m, \cdots, f$ are exponents of quantities, the range of each has maximum and minimum values; according to $^{8}$, integers are the following:

$$
\begin{aligned}
& -3 \leq l \leq+3,-1 \leq m \leq+1,-4 \leq t \leq+4,-2 \leq i \leq+2, \\
& -4 \leq \Theta \leq+4,-1 \leq j \leq+1,-1 \leq f \leq+1 ;
\end{aligned}
$$

(3) The exponents of quantities take only integer values [8], so the number of choices of dimensions for each quantity $e_{k},\{l, m, \cdots, f\}$ according to (3) is the following:

$$
e_{l}=7 ; e_{m}=3 ; e_{t}=9 ; e_{i}=5 ; e_{\theta}=9 ; e_{j}=3 ; e_{f}=3 ;
$$

(4) The total number of dimension options of physical quantities equals $\Psi^{*}=\prod_{l}^{f} e_{i}-1$

$$
\Psi^{*}=e_{l} \cdot e_{m} \cdot e_{t} \cdot e_{i} \cdot e_{\theta} \cdot e_{j} \cdot e_{f}-1=7 \times 3 \times 9 \times 5 \times 9 \times 3 \times 3-1=76544,
$$

where " -1 " corresponds to the situation when all exponents of base quantities in the formula (2) are treated as zero dimension; $\Pi$ is a product of $e_{k}$;

(5) The value $\Psi^{*}$ includes both required, and inverse quantities (for example, $L^{1}$-length, $L^{-1}$-running length). That is why the object can be judged knowing only one of its symmetrical parts, while others structurally duplicating this part may be regarded as information empty [9]. Therefore, the number of options of dimensions may be reduced by two times. It means that the total number of dimensional physical quantities without inverse quantities for SI equals

$$
\Psi=\Psi^{*} / 2=38272 ;
$$

(6) $z^{\prime}$ is the total number of dimensional physical quantities in the chosen class of phenomena $(C o P)$; in SI frames, every researcher selects a particular $C o P$ to study a material object. $C o P$ is a set of physical phenomena and processes described by a finite number of primary and secondary quantities that characterize certain features of a material object from the view of qualitative and quantitative aspects [10]. In studying mechanics, for example, which is widely applied for the Newtonian gravitational constant measurements with a torsion balance, the base units of SI are typically used: $L, M, T(L M T)$. In publications closed to studying, for example, the Boltzmann constant there was usually realized $C o P_{\mathrm{SI}} \equiv L M T \theta F$ and $C o P_{\mathrm{SI}} \equiv L M T \theta I$

(7) $\beta^{\prime}$ is the number of base physical quantities in the chosen $C o P$.

Taking into account the $\pi$-theorem [7], the total number of dimensionless criteria $\mu_{\mathrm{SI}}=\Psi-\xi$ inherent in SI equals 


$$
\mu_{\mathrm{SI}}=\Psi-\xi=38265 .
$$

Equation (1) quantifies $\Delta u / S$ caused by the limited number of quantities taken into account in the theoretical or experimental analysis of the researched quantity. On the other hand, it also sets a limit on the expedient increasing of the measurement accuracy in conducting experimental studies. In turn, $\Delta u / S$ is not a purely mathematical abstraction. It has a physical meaning, consisting in the observation that in nature there is a fundamental limit to the accuracy of displaying any observed material object, which cannot be surpassed by any improvement of instruments and methods of measurement. The reality of the environment is the obvious a priori condition for the modelling of the investigated material object. By allocating the interested process or phenomenon, the unknown relationships between the content of the object and the environment are "broken". In this context, it is obvious that an overall uncertainty of the model including inaccurate input data, physical assumptions, the approximate solution of the integral-differential equations, etc., will be larger than $\Delta u$. Thus, $\Delta u$ is only one lowest component of a possible mismatch of the real object and its modelling results.

In fact, Equation (1) can be regarded as the conformity principle (uncertainty relation) for the process of model development. No model can produce results that contradict relation (1). That is, any change in the level of the detailed description of the observed object $\left(z^{\prime \prime}-\beta^{\prime \prime} ; z^{\prime}-\beta^{\prime}\right)$ causes a change in the minimum comparative uncertainty value $\Delta u / S$ of the model of a specific $C o P$ and in the achieved accuracy of each main quantity, characterizing the internal structure of the object. In other words, the conformity principle fundamentally establishes the accuracy limit (for a given class of phenomena) of simultaneously defining a pair of quantities, observed by a conscious researcher, particularly, the absolute uncertainty in the measurement of the investigated quantity and the interval of its changes.

Equating the derivative of $\Delta u / S(1)$ with respect to $z^{\prime}-\beta^{\prime}$, to zero, we obtain the condition for achieving the minimum comparative uncertainty for a particular CoP:

$$
\left(z^{\prime}-\beta^{\prime}\right)^{2} /(\Psi-\xi)=\left(z^{\prime \prime}-\beta^{\prime \prime}\right)
$$

Table 1 introduces different classes of phenomena and the corresponding achievable comparative uncertainties and recommended number of quantities:

Let us apply the information approach for analysing measurement results of the Boltzmann constant by the use of four different methods.

For these purposes, the reader needs to remember that if the range of observation $S$ is not defined, the information obtained during the observation/measurement cannot be determined, and the entropic price becomes infinitely large [11]. Any specific measurement requires a finite a priori information about the components of the measurement and interval of observation of the measured quantity. These requirements are so universal that it acts as a postulate 
Table 1. Comparative uncertainties and recommended number of dimensionless criteria.

\begin{tabular}{|c|c|c|}
\hline$C o P_{\mathrm{SI}}$ & Comparative uncertainty & Number of criteria \\
\hline$L M T$ & 0.0048 & $0.2<1$ \\
\hline$L M T F$ & 0.0146 & $\cong 2$ \\
\hline$L M T I$ & 0.0245 & $\cong 6$ \\
\hline$L M T \theta$ & 0.0442 & $\cong 19$ \\
\hline$L M T I F$ & 0.0738 & $\cong 52$ \\
\hline$L M T \theta F$ & 0.1331 & $\cong 169$ \\
\hline$L M T \theta I$ & 0.2220 & $\cong 471$ \\
\hline$L M T \theta F I$ & 0.6665 & $\cong 4249$ \\
\hline
\end{tabular}

of metrology [12]. This, the observed range of variations, depends on the knowledge of the developer before undertaking the study. "If nothing is known about the system studied, then $S$ is determined by the limits of the measuring devices used" [11].

That is why, taking into account Brillouin's suggestions, there are two options for applying the conformity principle to analyse the measurement data of the fundamental physical constants.

First, this principle dictates, factually, analysing the data of the magnitude of the achievable relative uncertainty at the moment taking into account the latest results of measurements. The extended range of changes in the quantity under study $S$ indicates an imperfection of the measuring devices, which leads to a large value of the relative uncertainty. The development of measuring technology, the increase in the accuracy of measuring instruments, and the improvement in the existing and newly created measurement methods together lead to an increase in the knowledge of the object under study and, consequently, the magnitude of the achievable relative uncertainty decreases. However, this process is not infinite and is limited by the conformity principle. The reader should bear in mind that this conformity principle is not a shortcoming of the measurement equipment or engineering device but of the way the human brains work. When predicting the behaviour of any physical process, physicists are, in fact, predicting the perceivable output of instrumentation. It is true that, according to the $\mu$-hypothesis, observation is not a measurement but a process that creates a unique physical world with respect to each particular observer. Thus, in this case, the range of observation (possible interval of placing) of the fundamental physical constant $S$ is chosen as the difference between the maximum and minimum values of the physical constant measured by different scientific groups during a certain period of recent years. Only in the presence of the results of various experiments can one speak about the possible appearance of a measured value in a certain range. Thus, using the smallest attainable comparative uncertainty inherent in the selected class of phenomena during measuring the fundamental constant, it is possible to calculate the recommended minimum relative uncertainty 
that is compared with the relative uncertainty of each published study. In what follows, this method is denoted as IARU and includes the following steps:

1) From the published data of each experiment, the value $z$, relative uncertainty $r_{z}$ and standard uncertainty $u_{z}$ (possible interval of $u$ placing) of the fundamental physical constant are chosen;

2) The experimental absolute uncertainty $\Delta_{z}$ is calculated by multiplying the fundamental physical constant value $z$ and its relative uncertainty $r_{z}$ attained during the experiment, $\Delta_{z}=Z \cdot r_{z} ;$

3) The maximum $z_{\max }$ and minimum $z_{\min }$ values of the measured physical constant are selected from the list of measured values $Z_{i}$ of the fundamental physical constant obtained in different studies;

4) As a possible interval for placing the observed fundamental constant $S_{z z}$ the difference between the maximum and minimum values is calculated, $S_{z}=Z_{\max }-$ $Z_{\min }$

5) The selected comparative uncertainty $\mathcal{E}_{T}$ (Table 1 ) inherent in the model describing the measurement of the fundamental constant is multiplied by the possible interval of placement of the observed fundamental constant $S_{z}$ to obtain the absolute experimental uncertainty value $\Delta_{\text {IARU }}$ in accordance with the IARU, $\Delta_{\text {IARU }}=\varepsilon_{T^{\prime}} S_{z}$;

6) To calculate the relative uncertainty $r_{I A R U}$ in accordance with the $I A R U$, this absolute uncertainty $\Delta_{I A R U}$ is divided by the arithmetic mean of the selected maximum and minimum values, $r_{\text {IARU }}=\Delta_{\text {IARU }} /\left(\left(z_{\max }+z_{\min }\right) / 2\right)$;

7) The relative uncertainty obtained $r_{I A R U}$ is compared with the experimental relative uncertainties $r_{i}$ achieved in various studies;

8) According to $I A R U$, a comparative experimental uncertainty of each study, $\mathcal{E}_{I A R U i}$ is calculated by dividing the experimental absolute uncertainty of each study $\Delta_{z}$ by the difference between the maximum and minimum values of the measured fundamental constant $S_{z}, \varepsilon_{I A R U_{i}}=\Delta_{z} / S_{z}$. These calculated comparative uncertainties are also compared with the selected comparative uncertainty $\varepsilon_{T}$ (Table 1).

Second, $S$ is determined by the limits of the measuring devices used [11]. This means that as the observation interval in which the expected true value of the measured fundamental physical constant is located, a standard uncertainty is selected when measuring the physical constant in each particular experiment. Compared with various fields of technology, experimental physics is better for the fact that in all the researches, the experimenters introduce the output data of the measurement with uncertainty bars. At the same time, it should be remembered that the standard uncertainty of a particular measurement is subjective because the conscious observer probably did not take into account this or that uncertainty. The experimenters calculate the standard uncertainty, taking into account all possibilities, they noticed in the measured uncertainties. Then, one calculates the ratio between the absolute uncertainty reached in an experiment and the standard uncertainty, acting as a possible interval for allocating a fun- 
damental physical constant. So, in the framework of the information approach, the comparative uncertainties achieved in the studies are calculated and compared with the theoretically achievable comparative uncertainty inherent in the chosen class of phenomena. The standard uncertainty can be calculated also for quantities that are not normally distributed. Transformation of different types of uncertainty sources into standard uncertainty is very important. In what follows, this method is denoted as $I A C U$ and includes the following steps.

1) From the published data of each experiment, the value $z$, relative uncertainty $\mathrm{r}_{z}$ and standard uncertainty $u_{z}$ (possible interval of placing) of the fundamental physical constant are chosen;

2) The experimental absolute uncertainty $\Delta_{z}$ is calculated by multiplying the fundamental physical constant value $z$ and its relative uncertainty $r_{z}$ attained during the experiment, $\Delta_{z}=z \cdot r_{z} ;$

3) The achieved experimental comparative uncertainty of each published research $\varepsilon_{I A C U_{i}}$ is calculated by dividing the experimental absolute uncertainty $\Delta_{z}$ by the standard uncertainty $u_{z}, \mathcal{E}_{I A C U_{i}}=\Delta_{z} / u_{z}$;

4) The experimental calculated comparative uncertainty $\varepsilon_{I A C U i}$ is compared with the selected comparative uncertainty $\varepsilon_{\mathrm{T}}$ (Table 1 ) inherent in the model, which describes the measurement of the fundamental constant.

\section{Application of the Information Method}

\subsection{Acoustic Gas Thermometer}

First, confirm that you have the correct template for your paper size. This template has Acoustic gas thermometry (AGT) relates the speed of sound in a gas of known composition to the thermodynamic temperature. A detailed description of the design of the experimental method of measurement of the Boltzmann constant by the AGT method is presented in [13]. The measurement data are summarized in Table 2. The noted scientific articles belong to $\operatorname{CoP} P_{\mathrm{SI}} \equiv L M T \Theta F$ [14]-[20]. Although the authors of the research studies cited in these papers mentioned all the possible sources of uncertainty, the values of absolute and relative uncertainties can still differ by more than two times. And a similar situation exists in the spread of the values of comparative uncertainty.

Following the IARU method, one can discuss the order of the desired value of the relative uncertainty belonging to $C o P_{\mathrm{SI}} \equiv L M T \Theta F$. An estimated observation interval of $k$ is chosen as the difference in its values obtained from the experimental results of two projects: $k_{\max }=1.3806508 \times 10^{-23} \mathrm{~m}^{2} \cdot \mathrm{kg} \cdot \mathrm{s}^{-2} \cdot \mathrm{K}^{-1}$ [17] and $k_{\min }=1.3806484 \times 10^{-23} \mathrm{~m}^{2} \cdot \mathrm{kg} \cdot \mathrm{s}^{-2} \cdot \mathrm{K}^{-1}$ [20]. In this case, the possible observed range $S_{k}$ of $k$ placing is equal to:

$$
S_{k}=k_{\max }-k_{\min }=2.4 \times 10^{-29} \mathrm{~m}^{2} \cdot \mathrm{kg} /\left(\mathrm{s}^{2} \cdot \mathrm{K}\right)
$$

For this purpose, taking into account (4) and (8), one can arrive at the lowest comparative uncertainty $\mathcal{E}_{L M T \theta F}$ using the following conditions:

$$
\left(z^{\prime}-\beta^{\prime}\right)_{L M T \theta F}=\left(e_{l} \cdot e_{m} \cdot e_{t} \cdot e_{\theta} \cdot e_{f}-1\right) / 2-5=(7 \times 3 \times 9 \times 9 \times 3-1) / 2-5=2546
$$




$$
\left(z^{\prime \prime}-\beta^{\prime \prime}\right)_{L M T \theta F}=\left(z^{\prime}-\beta^{\prime}\right)^{2} / \mu_{S I}=2546^{2} / 38265 \approx 169
$$

where " -1 " corresponds to the case where all the base quantities exponents are zero in formula (1); 5 corresponds to the five base quantities $L, M, T, \Theta$ and $F$; and division by 2 indicates that there are direct and inverse quantities, e.g., $\mathrm{L}^{1}$ is the length and $\mathrm{L}^{-1}$ is the run length. The object can be judged based on the knowledge of only one of its symmetrical parts, while the other parts that structurally duplicate this one may be regarded as information empty. Therefore, the number of options of dimensions may be reduced by a factor of two.

According to (8), (10) and (11):

$$
\varepsilon_{L M T \theta F}=(\Delta u / S)_{L M T \theta F}=2546 / 38265+169.4 / 2546=0.1331
$$

Then, the lowest possible absolute uncertainty for $C o P_{\mathrm{SI}} \equiv L M T \Theta F$ is given by the following:

$$
\Delta_{\text {LMTAF }}=\varepsilon_{\text {LMTEF }} \cdot S_{k}=0.1331 \times 2.4 \times 10^{-29}=3.2 \times 10^{-30} \mathrm{~m}^{2} \cdot \mathrm{kg} /\left(\mathrm{s}^{2} \cdot \mathrm{K}\right)
$$

In this case, the lowest possible relative uncertainty $\left(r_{\min }\right)_{L M T \theta F}$ for $C o P_{\mathrm{SI}} \equiv$ $L M T \Theta F$ is the following:

$$
r_{L M T \theta F}=\Delta_{L M T \theta F} /\left(\left(k_{\max }+k_{\min }\right) / 2\right)=3.2 \times 10^{-30} / 1.38064961 \times 10^{-23}=2.3 \times 10^{-7}
$$

This value agrees well with [18] and the recommendation of $3.7 \times 10^{-7}$ cited in [21].

Guided by the IACU and IARU methods, one can calculate the achieved comparative uncertainty in each experiment (Table 2). There is a significant difference between the comparative uncertainty calculated according to the information-oriented approach $\varepsilon_{L M T \theta F}=0.1331$ and the experimental magnitudes achieved during measuring $k$. The difference may be explained by the following. AGT is based on the definition of an ideal gas behaviour in spite that the interaction between the gas particles is not sufficiently known. In addition, it is impossible to measure the molar concentration of gas per unit volume and volume itself with a competitive degree of accuracy. Moreover, the latter includes the volume of connecting pipes to pressure gauges. Thus, there are significant unrecorded uncertainties associated both with the formulation of the model of the experiment and with the achievable accuracy of the quantities taken into account in the calculation. It must be noted that the proximity of the acoustic mode to the shell resonance perturbs the data for this mode to an unacceptably large degree. In addition, experimenters take into account a very contrasting number of quantities in comparison to the recommendations (see Table 1). So, the significant difference between the comparative uncertainty calculated according to the information-oriented approach and the experimental magnitudes achieved during measuring $k$, confirms the observation that AGT has reached a limit that is unlikely to be improved for many years [23].

\subsection{Dielectric Constant Gas Thermometer (DCGT)}

In the DCGT, the gas dielectric constant $\varepsilon_{r}(p)$ is measured as a function of pres- 
sure to determine the temperature. To verify the Boltzmann constant, measurements are performed at a known temperature, the triple point of water (TTPW = $273.16 \mathrm{~K}$ ), and a $k$ value extracted from the Clausius-Mossotti ratio modified by the corresponding virial terms. The principle is quite simple: the capacitance $C(p)$ of a capacitor in a gas and its value in vacuum $C(0)$ are measured. Then, their ratio is calculated to extract the value of $\varepsilon_{r}$. The value of $k$ is then derived from $\varepsilon_{r}$ using the known molar polarizability, the density and dielectric virial coefficients [23].

The measurement data are summarized in Table 3. The noted scientific articles belong to $C o P_{\mathrm{SI}} \equiv L M T \Theta I[21]$ [24]-[28]. The values of absolute and relative uncertainties differ by more than a factor of 10. A similar situation exists in the spread of the values of comparative uncertainties.

Table 2. Determinations of the Boltzmann constant and achieved relative and comparative uncertainties using AGT.

\begin{tabular}{|c|c|c|c|c|c|c|c|c|}
\hline \multirow[t]{3}{*}{ Year } & \multirow[t]{3}{*}{$\mathrm{CoP}$} & \multirow{3}{*}{$\begin{array}{c}\begin{array}{c}\text { Boltzmann's } \\
\text { constant }\end{array} \\
k \cdot 10^{23} \\
\mathrm{~m}^{2} \mathrm{~kg} /\left(\mathrm{s}^{2} \mathrm{~K}\right)\end{array}$} & $\begin{array}{c}\text { Achieved } \\
\text { relative } \\
\text { uncertainty }\end{array}$ & $\begin{array}{c}\text { Absolute } \\
\text { uncertainty }\end{array}$ & $\begin{array}{c}\boldsymbol{k}_{\mathrm{b}} \text { possible } \\
\text { interval of } \\
\text { placing }^{*}\end{array}$ & $\begin{array}{l}\text { Calculated } \\
\text { comparative } \\
\text { uncertainty }\end{array}$ & $\begin{array}{l}\text { Calculated } \\
\text { comparative } \\
\text { uncertainty }\end{array}$ & Ref. \\
\hline & & & \multirow{2}{*}{$\mathrm{r}_{k} \cdot 10^{6}$} & $\Delta_{k} \cdot 10^{29}$ & $\mathrm{u}_{k} \cdot 10^{29}$ & \multirow{2}{*}{$\begin{array}{c}E_{k}^{\prime}=\Delta_{k} / \mathrm{u}_{k} \\
I A C U\end{array}$} & \multirow{2}{*}{\multicolumn{2}{|c|}{$\begin{array}{c}\mathcal{E}_{k}^{\prime \prime}=\Delta_{k} / S_{k} \\
I A R U\end{array}$}} \\
\hline & & & & \multicolumn{2}{|c|}{$\overline{\mathrm{m}^{2} \mathrm{~kg} /\left(\mathrm{s}^{2} \mathrm{~K}\right) \mathrm{m}^{2} \mathrm{~kg} /\left(\mathrm{s}^{2} \mathrm{~K}\right)}$} & & & \\
\hline 2009 & & 1.3806495 & 2.7 & 3.73 & 7.4 & 0.5038 & 1.1393 & [14] \\
\hline 2010 & & 1.3806496 & 3.1 & 4.28 & 8.8 & 0.4864 & 1.3081 & [15] \\
\hline 2015 & & 1.3806487 & 2.0 & 2.76 & 2.7 & 1.0227 & 0.8439 & [16] \\
\hline 2015 & $L M T \Theta F$ & 1.3806508 & 1.1 & 1.52 & 2.9 & 0.5237 & 0.4642 & [17] \\
\hline 2017 & & 1.3806488 & 0.6 & 0.83 & 1.6 & 0.5177 & 0.2532 & [18] \\
\hline 2017 & & 1.3806486 & 0.7 & 0.97 & 2.0 & 0.4832 & 0.2954 & [19] \\
\hline 2017 & & 1.3806484 & 2.0 & 2.76 & 5.5 & 0.5020 & 0.8439 & [20] \\
\hline
\end{tabular}

* Data are introduced in [21] [22] [23].

Table 3. Determinations of the Boltzmann constant and achieved relative and comparative uncertainties using DCGT.

\begin{tabular}{|c|c|c|c|c|c|c|c|c|}
\hline \multirow{3}{*}{ Year } & \multirow{3}{*}{$\mathrm{CoP}$} & $\begin{array}{c}\text { Boltzmann's } \\
\text { constant }\end{array}$ & $\begin{array}{c}\text { Achieved } \\
\text { relative } \\
\text { uncertainty }\end{array}$ & $\begin{array}{c}\text { Absolute } \\
\text { uncertainty }\end{array}$ & $\begin{array}{c}\boldsymbol{k}_{\mathrm{b}} \text { possible } \\
\text { interval of } \\
\text { placing* }^{*}\end{array}$ & $\begin{array}{l}\text { Calculated } \\
\text { comparative } \\
\text { uncertainty }\end{array}$ & $\begin{array}{l}\text { Calculated } \\
\text { comparative } \\
\text { uncertainty }\end{array}$ & \multirow{3}{*}{ Ref. } \\
\hline & & $k \cdot 10^{23}$ & \multirow[b]{2}{*}{$\mathrm{r}_{k} \cdot 10^{6}$} & $\Delta_{k} \cdot 10^{29}$ & $\mathrm{u}_{k} \cdot 10^{29}$ & \multirow{2}{*}{$\begin{array}{c}\varepsilon_{k}^{\prime}=\Delta_{k} / \mathrm{u}_{k} \\
I A C U\end{array}$} & \multirow{2}{*}{$\begin{array}{c}\mathcal{E}_{k}^{\prime \prime}=\Delta_{k} / S_{k} \\
I A R U\end{array}$} & \\
\hline & & $\mathrm{m}^{2} \mathrm{~kg} /\left(\mathrm{s}^{2} \mathrm{~K}\right)$ & & $\begin{array}{c}\mathrm{m}^{2} \mathrm{~kg} / \\
\left(\mathrm{s}^{2} \mathrm{~K}\right)\end{array}$ & $\begin{array}{c}\mathrm{m}^{2} \mathrm{~kg} / \\
\left(\mathrm{s}^{2} \mathrm{~K}\right)\end{array}$ & & & \\
\hline 2012 & \multirow{6}{*}{$L M T \Theta I$} & 1.38066509 & 7.9 & 1.09 & 6.7 & 1.6279 & 4.5983 & [24] \\
\hline 2013 & & 1.38066509 & 4.3 & 5.94 & 1.2 & 4.9473 & 2.5029 & [25] \\
\hline 2015 & & 1.38066509 & 4.0 & 5.52 & 11.0 & 0.5020 & 2.3282 & [26] \\
\hline 2017 & & 1.3806482 & 1.9 & 2.62 & 5.2 & 0.5044 & 1.1059 & [27] \\
\hline 2018 & & 1.38064904 & 0.37 & 0.51 & 1.0 & 0.5184 & 0.2154 & [21] \\
\hline 2018 & & 1.3806490 & 0.37 & 0.51 & 1.0 & 0.5184 & 0.2154 & [28] \\
\hline
\end{tabular}

* Data are introduced in [13] [28]. 
Following the method IARU, one can discuss the order of the desired value of the relative uncertainty belonging to $C o P_{\mathrm{SI}} \equiv L M T \Theta I$. An estimated observation interval of $k$ is chosen as the difference in its values obtained from the experimental results of two projects: $k_{\max }=1.38066509 \times 10^{-23} \mathrm{~m}^{2} \cdot \mathrm{kg} \cdot \mathrm{s}^{-2} \cdot \mathrm{K}^{-1}$ [26] and $k_{\min }=1.3806482 \times 10^{-23} \mathrm{~m}^{2} \cdot \mathrm{kg} \cdot \mathrm{s}^{-2} \cdot \mathrm{K}^{-1}$ [27]. In this case, the possible observed range $S_{k}$ of $k$ placing is equal to:

$$
S_{k}=k_{\max }-k_{\min }=2.7 \times 10^{-29} \mathrm{~m}^{2} \cdot \mathrm{kg} /\left(\mathrm{s}^{2} \cdot \mathrm{K}\right)
$$

For this purpose, taking into account (4) and (8), one can arrive at the lowest comparative uncertainty $\mathcal{E}_{L M T \theta I}$ using the following conditions:

$$
\begin{gathered}
\left(z^{\prime}-\beta^{\prime}\right)_{\text {LMTOI}}=\left(e_{l} \cdot e_{m} \cdot e_{t} \cdot e_{\theta} \cdot e_{i}-1\right) / 2-5=(7 \times 3 \times 9 \times 9 \times 5-1) / 2-5=4247 \\
\left(z^{\prime \prime}-\beta^{\prime \prime}\right)_{L M T \theta I}=\left(z^{\prime}-\beta^{\prime}\right)^{2} / \mu_{S I}=4247^{2} / 38265 \approx 471
\end{gathered}
$$

where " -1 " corresponds to the case where all the base quantities exponents are zero in formula (1); 5 corresponds to the five base quantities $L, M, T, \Theta$ and $I$; and division by 2 indicates that there are direct and inverse quantities, e.g., $\mathrm{L}^{1}$ is the length and $\mathrm{L}^{-1}$ is the run length. The object can be judged based on the knowledge of only one of its symmetrical parts, while the other parts that structurally duplicate this one may be regarded as information empty. Therefore, the number of options of dimensions may be reduced by a factor of two.

According to (16) and (17):

$$
\varepsilon_{\text {LMTEI }}=(\Delta u / S)_{\text {LMTEI }}=4247 / 38265+471 / 4247=0.222
$$

Taking into account (18), the lowest possible absolute uncertainty for DCGT $\left(C o P_{\mathrm{SI}} \equiv L M T \Theta I\right)$ is given by the following:

$$
\Delta_{\text {LMTEI }}=\varepsilon_{L M T \theta I} \cdot S_{k}=0.222 \times 2.7 \times 10^{-29}=6.0 \times 10^{-30} \mathrm{~m}^{2} \cdot \mathrm{kg} /\left(\mathrm{s}^{2} \cdot \mathrm{K}\right)
$$

In this case, the lowest possible relative uncertainty $\left(r_{\min }\right)_{L M T \theta I}$ for DCGT and the achieved mentioned results is the following:

$$
r_{\text {LMTEI }}=\Delta_{\text {LMTEI }} /\left(\left(k_{\max }+k_{\min }\right) / 2\right)=6.0 \times 10^{-30} / 1.38064955 \times 10^{-23}=4.3 \times 10^{-7}
$$

This value is in a strong contradiction with the recommendation of $3.7 \times 10^{-7}$ cited in [21]. In the frame of the information approach, any experimental values of the relative uncertainty must be greater than the relative uncertainty corresponding to the DCGT method ( $\left.C o P_{\mathrm{SI}} \equiv L M T \Theta I\right)$, i.e. $4.3 \times 10^{-7}$. In other words, there are unrecorded uncertainties in [21], and therefore, experimenters are advised to double-check the full list of sources of uncertainty taken into account.

Guided by the IACU and IARU methods, one can calculate the achieved comparative uncertainty in each experiment (Table 3 ). There is a large gap between the comparative uncertainty calculated according to the information-oriented approach $\varepsilon_{L M T \theta I}=0.222$ and the experimental magnitudes achieved during measuring $k$ by DCGT. At the same time, progress to achieving a higher accuracy is obvious during the last four years.

Significant differences in the values of the comparative uncertainties achieved 
in the experiments and calculated in accordance with the $I A C U$ can be explained as follows. Within the framework of the information approach, the concept of comparative uncertainty assumes an equally probable account of various quantities, regardless of their specific choice by scientists when formulating a model for measuring $k$. Based on their experience, intuition and knowledge, the researchers build a model containing a small number of quantities, and which, in their opinion, reflects the fundamental essence of the process under investigation. In this case, many phenomena, perhaps not significant, secondary, which are characterized by specific quantities, are not taken into account.

For example, in DCGT, the measuring gas (helium) has very low polarizability, which prevents absolute measurements of a dielectric constant $\mathcal{E}$. It is calculated from the relative change in capacitance of a capacitor. Because of the necessary hydrostatic correction, pressure measurement is a limiting factor, besides dielectric virial corrections and compressibility problems. During the measurement, pairs of values of the pressure $p$ and of the dielectric constant $\varepsilon$ are recorded at a temperature which must be constant, however, very small fluctuations exists. $k$ is determined from the function $\varepsilon(p)$, a component of which must be linearly approximated. In addition, the applied high gas pressures cause a deformation of the measuring capacitors. Thus, there are significant unrecorded uncertainties associated both with the formulation of the model of the experiment and with the achievable accuracy of the quantities taken into account in the calculation. Besides, experimenters take into account a very contrasting number of quantities in comparison to the recommendations (see Table 1). So, the lack of previous experience influences innovative research, although specialists are highly qualified to use the latest technologies. Thus, a paradoxical situation arises when new inconsistencies give rise to more serious vulnerabilities in new measurement and computing technologies [29]. In this situation, a new information approach can play a positive role in predicting and adopting new definitions of units for the international SI system.

\subsection{Johnson Noise Thermometer}

In the JNT technique a well-characterized noble gas and pressure measurements are used at the frontier of accuracy, as well as primary level electrical metrology to measure minute changes in tiny capacitances $(\approx 10 \mathrm{pF})$. Thermodynamic temperature is derived from the statistical movement of electrons in an ohmic resistor, generating a noise voltage (white noise). JNT determines the temperature from the mean square of the noise voltage by means of the Nyquist formula [13].

The measurement data are summarized in Table 4. The noted scientific articles belong to $\operatorname{CoP} P_{\mathrm{SI}} \equiv L M T \Theta I$ [30]-[35]. The values of absolute and relative uncertainties differ by more than a factor of five. A similar situation exists in the spread of the values of comparative uncertainties.

Following the method IARU, one can discuss the order of the desired value of the relative uncertainty belonging to $C o P_{\mathrm{SI}} \equiv L M T \Theta I$. An estimated observation 
Table 4. Determinations of the Boltzmann constant and achieved relative and comparative uncertainties using JNT.

\begin{tabular}{|c|c|c|c|c|c|c|}
\hline \multirow{3}{*}{ Year } & \multirow{3}{*}{$\mathrm{CoP}$} & $\begin{array}{c}\text { Boltzmann's } \\
\text { constant }\end{array}$ & $\begin{array}{l}\text { Achieved } \\
\text { relative } \\
\text { uncertainty }\end{array}$ & $\begin{array}{l}\text { Absolute } \\
\text { uncertainty }\end{array}$ & $\begin{array}{l}\text { Calculated } \\
\text { comparative } \\
\text { uncertainty* }\end{array}$ & \multirow{3}{*}{ Ref. } \\
\hline & & $k \cdot 10^{23}$ & \multirow{2}{*}{$\mathrm{r}_{k} \cdot 10^{6}$} & $\Delta_{k} \cdot 10^{29}$ & \multirow{2}{*}{$\begin{array}{c}\varepsilon_{k}^{\prime \prime}=\Delta_{k} / S_{k} \\
I A R U\end{array}$} & \\
\hline & & $\mathrm{m}^{2} \mathrm{~kg} /\left(\mathrm{s}^{2} \mathrm{~K}\right)$ & & $\mathrm{m}^{2} \mathrm{~kg} /\left(\mathrm{s}^{2} \mathrm{~K}\right)$ & & \\
\hline 2011 & \multirow{6}{*}{$L M T \Theta I$} & 1.38065200 & 12.0 & 16.7 & 7.0430 & [30] \\
\hline 2015 & & 1.38065160 & 3.9 & 5.4 & 2.2700 & [31] \\
\hline 2015 & & 1.38064974 & 71.0 & 98.0 & 41.326 & [32] \\
\hline 2017 & & 1.38064974 & 2.7 & 3.7 & 1.5716 & [33] \\
\hline 2017 & & 1.38064300 & 5.0 & 6.9 & 2.9103 & [34] \\
\hline 2017 & & 1.38064360 & 10.0 & 14.1 & 5.9370 & [35] \\
\hline
\end{tabular}

* No summarized data about a value of the standard uncertainty required for calculation of the comparative uncertainty by $I A C U$.

interval of $k$ is chosen as the difference in its values obtained from the experimental results of two projects: $k_{\max }=1.38065200 \times 10^{-23} \mathrm{~m}^{2} \cdot \mathrm{kg} \cdot \mathrm{s}^{-2} \cdot \mathrm{K}^{-1}[30]$ and $k_{\min }=1.38064300 \times 10^{-23} \mathrm{~m}^{2} \cdot \mathrm{kg} \cdot \mathrm{s}^{-2} \cdot \mathrm{K}^{-1}$ [34]. In this case, the possible observed range $S_{k}$ of $k$ placing is equal to:

$$
S_{k}=k_{\max }-k_{\min }=9.0 \times 10^{-29} \mathrm{~m}^{2} \cdot \mathrm{kg} /\left(\mathrm{s}^{2} \cdot \mathrm{K}\right)
$$

According to (16) and (17):

$$
\varepsilon_{\text {LMTөI }}=(\Delta u / S)_{\text {LMTөI }}=4247 / 38265+471 / 4247=0.222
$$

Taking into account (23), the lowest possible absolute uncertainty for JNT $\left(C o P_{\mathrm{SI}} \equiv L M T \Theta I\right)$ is given by the following:

$$
\Delta_{\text {LMT } \theta I}=\varepsilon_{\text {LMTEI }} \cdot S_{k}=0.222 \times 9.0 \times 10^{-29}=2.0 \times 10^{-29} \mathrm{~m}^{2} \cdot \mathrm{kg} /\left(\mathrm{s}^{2} \cdot \mathrm{K}\right)
$$

In this case, the lowest possible relative uncertainty $\left(r_{\min }\right)_{\text {LMTOI }}$ for JNT and the achieved mentioned results is the following:

$$
r_{\text {LMTEI }}=\Delta_{\text {LMTEI }} /\left(\left(k_{\max }+k_{\min }\right) / 2\right)=2.0 \times 10^{-29} / 1.3806475 \times 10^{-23}=1.4 \times 10^{-6}
$$

This value agrees well with [33]. Guided by the IARU method, one can calculate the achieved comparative uncertainty in each experiment (Table 4). There is a large gap between the comparative uncertainty calculated according to the information-oriented approach $\varepsilon_{L M T \theta I}=0.222$ and the experimental magnitudes achieved during measuring $k$ by JNT. Unfortunately, there has been no progress to achieve higher accuracy during the last eight years.

There are obvious problems that can apparently be solved: a long measurement time required for low uncertainty. To achieve a relative uncertainty in the range of $1 \times 10^{-5}$ with a normal detection bandwidth of $20 \mathrm{kHz}$, a measurement time of about 5 weeks is required, during which unpredictable voltage surges in the network are possible. In addition, because of the inconsistency between the 
transfer lines of the thermal resistor and of the reference noise source, the experimental ratio spectrum varies with frequency. Therefore, the power spectral density ratio of the noise of the resistor to the noise of the quantum voltage must be simulated, causing another significant source of error. Progress in reducing the uncertainty of these factors suggests that a high potential of this method is possible when measuring the Boltzmann constant.

\subsection{Doppler Broadening Thermometer}

The DBT method uses the measurement of the Doppler width of the spectral line in a gas at a known temperature to determine the value of the Boltzmann constant. All experiments consist in measuring the Doppler width for a sample maintained at a temperature very close to TTPW. In principle, many different atoms or molecules can be used for the Doppler method of thermometry. Small, simple, non-polar, seemingly the best choice: the lighter the mass, the greater the Doppler width at a given temperature; in addition, lighter molecules have more widely separated vibrational transitions than heavy molecules. Non-polar molecules $\left(\mathrm{O}_{2}, \mathrm{CO}_{2}, \mathrm{C}_{2} \mathrm{H}_{2}\right)$ have lower collisional self-expansion than polar ones. Ultimately, the choices made by groups measuring the Boltzmann constant were often based on history, previous knowledge of the molecular structure, and equipment developed at the appropriate wavelength [23]. The results are shown in Table 5 [36]-[41].

Following the method IARU, one can discuss the order of the desired value of the relative uncertainty belonging to $C o P_{\mathrm{SI}} \equiv L M T \Theta F$. An estimated observation interval of $k$ is chosen as the difference in its values obtained from the experimental results of two projects: $k_{\max }=1.380802 \times 10^{-23} \mathrm{~m}^{2} \cdot \mathrm{kg} \cdot \mathrm{s}^{-2} \cdot \mathrm{K}^{-1}$ [38] and $k_{\min }$ $=1.3805813 \times 10^{-23} \mathrm{~m}^{2} \cdot \mathrm{kg} \cdot \mathrm{s}^{-2} \cdot \mathrm{K}^{-1}$ [39]. In this case, the possible observed range $S_{k}$ of $k$ placing is equal to:

$$
S_{k}=k_{\max }-k_{\min }=2.2 \times 10^{-27} \mathrm{~m}^{2} \cdot \mathrm{kg} /\left(\mathrm{s}^{2} \cdot \mathrm{K}\right)
$$

Taking into account (25), the lowest possible absolute uncertainty for DBT $\left(C o P_{\mathrm{SI}} \equiv L M T \Theta F\right)$ is given by the following:

$$
\Delta_{\text {LMTEF }}=\varepsilon_{\text {LMTEF }} \cdot S_{k}=0.222 \times 2.2 \times 10^{-27}=2.9 \times 10^{-28} \mathrm{~m}^{2} \cdot \mathrm{kg} /\left(\mathrm{s}^{2} \cdot \mathrm{K}\right)
$$

In this case, the lowest possible relative uncertainty $\left(r_{\min }\right)_{L M T \theta I}$ for DBT and the achieved mentioned result is the following:

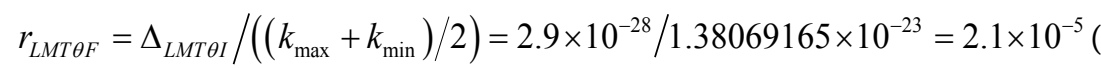

This value agrees well with [41]. Using the IARU method, it is possible to calculate the achieved comparative uncertainty in each experiment (Table 5). There is a large gap between the comparative uncertainty calculated in accordance with the information approach $\varepsilon_{L M T \theta I}=0.222$ and the experimental values achieved by measuring $k$ with the DBT method. However, sufficient progress to achieve higher accuracy in the last eight years is obvious. To improve the accuracy of measurements, it is necessary to solve the following problems. 
Table 5. Determinations of the Boltzmann constant and achieved relative and comparative uncertainties using DBT.

\begin{tabular}{|c|c|c|c|c|c|c|}
\hline \multirow{3}{*}{ Year } & \multirow{3}{*}{$\mathrm{CoP}$} & $\begin{array}{c}\text { Boltzmann's } \\
\text { constant }\end{array}$ & $\begin{array}{l}\text { Achieved } \\
\text { relative } \\
\text { uncertainty }\end{array}$ & $\begin{array}{c}\text { Absolute } \\
\text { uncertainty }\end{array}$ & $\begin{array}{l}\text { Calculated } \\
\text { comparative } \\
\text { uncertainty }\end{array}$ & \multirow{3}{*}{ Ref. } \\
\hline & & $k \cdot 10^{23}$ & \multirow{2}{*}{$\mathrm{r}_{k} \cdot 10^{5}$} & $\Delta_{k} \cdot 10^{28}$ & \multirow{2}{*}{$\begin{array}{c}\varepsilon_{k}^{\prime \prime}=\Delta_{k} / \mathrm{S}_{k} \\
I A R U\end{array}$} & \\
\hline & & $\mathrm{m}^{2} \mathrm{~kg} /\left(\mathrm{s}^{2} \mathrm{~K}\right)$ & & $\mathrm{m}^{2} \mathrm{~kg} /\left(\mathrm{s}^{2} \mathrm{~K}\right)$ & & \\
\hline 2007 & \multirow{6}{*}{$L M T \Theta F$} & 1.38065260 & 19.0 & 26.2 & 110.59 & [36] \\
\hline 2008 & & 1.38066952 & 3.8 & 5.2 & 22.119 & {$[37]$} \\
\hline 2009 & & 1.38080200 & 14.0 & 19.9 & 83.826 & {$[38]$} \\
\hline 2011 & & 1.38058130 & 16.0 & 22.1 & 93.125 & [39] \\
\hline 2014 & & 1.38066120 & 8.7 & 12.0 & 50.640 & {$[40]$} \\
\hline 2015 & & 1.38066313 & 2.4 & 3.3 & 13.969 & [41] \\
\hline
\end{tabular}

* No summarized data about a value of the standard uncertainty required for calculation of the comparative uncertainty by $I A C U$.

In DBT, the shape of the absorption line is not a purely Gaussian profile due to collisional broadening (the natural width of the line due to spontaneous emission is usually negligible). For polyatomic molecules, such as water and ammonia, used to measure $k$, the pressure-dependent broadening coefficients cannot be calculated at the beginning. In addition, line shapes are analysed using more or less complex models, none of which describe ideally the interaction of light and matter. The validity of such models is a stumbling block for higher accuracy. In this respect, atomic helium has non-ideal properties. Although its collision broadening coefficients can be calculated with slight uncertainty, helium resonance lines lie in the vacuum ultraviolet range, where there are no sources of continuous laser waves yet. Even in this case, besides fragile window cells and detectors, photoionization of excited particles will undoubtedly complicate the analysis of the line shape [23].

\section{Discussion}

The reality is not so simple. Estimates and measurements always have uncertainties. This concept is absolutely important for scientific research: what you know is far less important than how well you know it. The real main idea of any scientific discussion is to delve into uncertainties and how they are evaluated.

As mentioned above, to estimate the accuracy achieved in various natural and technological processes, including measurements of fundamental physical constants, the concept of relative uncertainty is used. This method of determining the measurement accuracy does not indicate the direction in which the true value of the fundamental physical constant can be found. In addition, it includes an element of subjective judgement [3].

Under the proposed approach, for each mathematical model of a physical law there is an uncertainty, which initially, before the full-scale experimental studies, 
or computer simulations, describes its proximity to the examined physical phenomenon or process. This value is called the comparative uncertainty. It depends only on the number of selected quantities and the observation interval of the selected primary quantity. One of the interesting features of the proposed hypothesis is that the minimum achievable comparative uncertainty is not constant and varies depending on the class of phenomena choice. Moreover, theory can predict its value. In particular, this means that when switching from a mechanistic model ( $L M T)$ to $C o P_{\mathrm{SI}}$ with a larger number of the base quantities, this uncertainty grows. This change is due to the potential effects of the interaction between the increased number of quantities that can be taken into account or not taken into account by the researcher. That is why, within the framework of the information approach, in contradiction with the concept approved by CODATA, it is not recommended to determine and declare only one value of relative uncertainty when measuring the Boltzmann constant by different methods.

In addition to the comments made in Section 3 regarding the analysis of the measurement results of the Boltzmann constant based on four different methods using IACU and IARU (summarized in Table 6), the following should be noted.

The greatest success in achieving high accuracy in measuring $k$ was achieved using DCGT, given the significant difference in the magnitude of the comparative uncertainties between $C o P_{\mathrm{SI}} \equiv L M T \Theta F(\mathrm{AGT}-0.1331)$ and $C o P_{\mathrm{SI}} \equiv L M T \Theta I$ (DCGT - 0.2220). However, experimenters involved in DCGT will have to carefully check all sources of error. This is due to the requirement of the information method, according to which the experimental relative uncertainty is always greater than the relative uncertainty, theoretically calculated.

At the moment, the DBT method seems to be very limited (in terms of its physical acceptability for measuring $k$ ) in the possibility of achieving higher accuracy. This is explained by the fact that the values of relative uncertainty, calculated according to $C o P_{\mathrm{SI}} \equiv L M T \Theta F$ and achieved in the experiment, are very close $\left(2.1 \times 10^{-5}\right.$ and $\left.2.4 \times 10^{-5}\right)$.

Table 6. Summarized data.

\begin{tabular}{|c|c|c|c|c|}
\hline Variable & AGT & DCGT & JNT & DBT \\
\hline $\mathrm{CoP}$ & $L M T \theta F$ & $L M T \theta I$ & $L M T \theta I$ & $L M T \theta F$ \\
\hline $\begin{array}{l}\text { Comparative uncertainty } \\
\text { according to } C o P_{\mathrm{SI}}\end{array}$ & 0.1331 & 0.2220 & 0.2220 & 0.1331 \\
\hline $\begin{array}{c}\mathrm{S}_{k}=\boldsymbol{k}_{\max }-\boldsymbol{k}_{\min } \\
\mathrm{m}^{2} \mathrm{~kg} /\left(\mathrm{s}^{2} \mathrm{~K}\right)\end{array}$ & $2.4 \times 10^{-29}$ & $2.7 \times 10^{-29}$ & $9.2 \times 10^{-29}$ & $2.2 \times 10^{-27}$ \\
\hline $\begin{array}{l}\text { Relative uncertainty } \\
\text { according to } C o P_{\mathrm{SI}}\end{array}$ & $2.3 \times 10^{-7}$ & $4.3 \times 10^{-7}$ & $1.4 \times 10^{-6}$ & $2.1 \times 10^{-5}$ \\
\hline $\begin{array}{l}\text { Achieved experimental } \\
\text { lowest relative uncertainty }\end{array}$ & $6.0 \times 10^{-7}$ & $3.7 \times 10^{-7}$ & $2.7 \times 10^{-6}$ & $2.4 \times 10^{-5}$ \\
\hline
\end{tabular}


The data on the JNT method, when compared with the DCGT method, make it possible to assert the possibility of achieving higher measurement accuracy $k$, given the large gap between the relative uncertainty calculated according to $C o P$ ${ }_{\mathrm{SI}} \equiv \operatorname{LMT\theta I}\left(1.4 \times 10^{-6}\right)$ and experimentally achieved $\left(2.7 \times 10^{-6}\right)$.

It would seem that the significant difference between the theoretical value of the relative uncertainty for AGT $\left(2.3 \times 10^{-7}\right)$ and the achieved $\left(6.0 \times 10^{-7}\right)$ allows hope for its constantly decreasing value when using this method. However, given the fact that the interval of possible placement of $k$ for the AGT method $(2.4 \times$ $\left.10^{-29} \mathrm{~m}^{2} \cdot \mathrm{kg} /\left(\mathrm{s}^{2} \cdot \mathrm{K}\right)\right)$ is the smallest compared with other methods, it is difficult to expect any achievements in increasing its accuracy.

\section{Conclusions}

In addition to analysing the relative uncertainty, the introduced approach may allow a new methodology that will help further monitor the accuracy of the Boltzmann constant measurement. The use of the $\mu_{\mathrm{SI}}$-hypothesis only limits the scope of the theory of measurements for uncertainties that are much larger than the model uncertainty due to its finiteness.

The $\mu_{\mathrm{SI}}$-hypothesis might be applicable to experimental verification. In general, it is available when the researcher has all the information about the uncertainty interval of the main quantity.

Because the proposed method is not associated with a specific structure of the model, which may change, it is more common, easy and leads to the solution of specific problems, without requiring detailed information about the set of quantities. However, it does not disclose the internal mechanism of phenomena.

Equation (1) clearly indicates that it is impossible to develop an experimental device that would allow achieving the exact value of the chosen comparative uncertainty for a given measurement result. The $\mu_{\mathrm{SI}}$-hypothesis, given in equation (1), makes the lower limit of the entropy change of the selected model inaccessible from theoretical considerations. This trend is reflected in the gap between the value of the calculated experimental comparative uncertainty and its theoretical lower limit, the value of which depends on the selected class of phenomena.

The information-oriented approach, in particular IARU, allows calculation of the relative uncertainty, which is in good agreement with the CODATA recommendations. The principal difference between this method and the existing statistical and expert CODATA methodology is the fact that the information method is theoretically justified. Thus, the presented method can serve as a practical tool for ascertaining the preferences, advantages and disadvantages of various methods of measuring the Boltzmann constant.

\section{Acknowledgements}

The author is grateful to engineer B. A. Alexandrov for his advice, fruitful discussions and who took the time to comment on previous drafts of this article. 


\section{Conflicts of Interest}

The author declares no conflicts of interest regarding the publication of this paper.

\section{References}

[1] BIPM, Consultative Committee for Thermometry (CCT) (2014) Report of the 27th meeting, Recommendation CCT T1. http://www.bipm.org/utils/common/pdf/CC/CCT/CCT27.pdf

[2] Dodson, B. (2013) Quantum Physics and the Nature of Reality (QPNR) Survey: 2011.

[3] Henrion, M. and Fischhoff, B. (1986) Assessing Uncertainty in Physical Constants. American Journal of Physics, 54, 791-798. https://doi.org/10.1119/1.14447

[4] Menin, B. (2018) h, k, NA: Evaluating the Relative Uncertainty of Measurement. American Journal of Computational and Applied Mathematics, 8, 93-102. http://article.sapub.org/10.5923.j.ajcam.20180805.02.html

[5] Menin, B. (2019) A Look at the Uncertainty of Measuring the Fundamental Constants and the Maxwell Demon from the Perspective of the Information Approach. Global Journal of Researchers in Engineering: A Mechanical and Mechanics Engineering, 19, 1-17.

https://globaljournals.org/GJRE_Volume19/1-A-Look-at-the-Uncertainty.pdf

[6] Menin, B. (2017) Information Measure Approach for Calculating Model Uncertainty of Physical Phenomena. American Journal of Computational and Applied Mathematics, 7, 11-24.

[7] Sonin, A.A. (2001) The Physical Basis of Dimensional Analysis. 2nd Edition, Department of Mechanical Engineering, MIT, Cambridge.

[8] NIST Special Publication 330 (SP330) (2008) The International System of Units (SI).

[9] Jakulin, A. (2004) Symmetry and Information Theory, 1-20.

[10] Sedov, L.I. (1993) Similarity and Dimensional Methods in Mechanics. CRC Press, Florida.

[11] Brillouin, L. (1964) Scientific Uncertainty and Information, Academic Press, New York.

[12] Balalaev, V.A., Slayev, V.A. and Sinyakov, A.I., (2005) Potential Accuracy of Measurements. Slayev, V.A., Ed., ANO NPO Professional, St. Petersburg. (In Russian) http://www.vniim.ru/files/PotTochIzm.pdf

[13] Fischer, J. (2018) The Boltzmann Constant for the Definition and Realization of the Kelvin. Annals of Physics (Berlin), Article ID: 1800304, 1-12. https://doi.org/10.1002/andp.201800304

[14] Pitre, L., et al. (2009) An Improved Acoustic Method for the Determination of the Boltzmann Constant at LNE-INM/CNAM. C. R. Physique, 10, 835-848. https://doi.org/10.1016/j.crhy.2009.11.001

[15] Sutton, G., Underwood, R., Pitre, L., de Podesta, M. and Valkiers, S. (2010) Acoustic Resonator Experiments at the Triple Point of Water: First Results for the Boltzmann Constant and Remaining Challenges. International Journal of Thermophysics, 31, 1310-1346. https://doi.org/10.1007/s10765-010-0722-Z

[16] Pitre, L. (2015) Determination of the Boltzmann Constant k from the Speed of Sound in Helium Gas at the Triple Point of Water. Metrologia, 52, 263-273. 
http://sci-hub.tw/10.1088/0026-1394/52/5/S263 https://doi.org/10.1088/0026-1394/52/5/S263

[17] Gavioso, R.M. (2015) A Determination of the Molar Gas constant R by Acoustic Thermometry in Helium. Metrologia, 52, 274-304.

http://sci-hub.tw/10.1088/0026-1394/52/5/S274 https://doi.org/10.1088/0026-1394/52/5/S274

[18] Pitre, L., et al. (2017) New Measurement of the Boltzmann Constant k by Acoustic Thermometry of Helium-4 Gas. Metrologia, 54, 856-873.

https://ws680.nist.gov/publication/get_pdf.cfm?pub_id=923465 https://doi.org/10.1088/1681-7575/aa7bf5

[19] de Podesta, M., et al. (2017) Re-Estimation of Argon Isotope Ratios Leading to a Revised Estimate of the Boltzmann Constant. Metrologia, 54, 683-692.

http://sci-hub.tw/10.1088/1681-7575/aa7880 https://doi.org/10.1088/1681-7575/aa7880

[20] Feng, X.J., et al. (2017) Determination of the Boltzmann Constant with Cylindrical Acoustic Gas Thermometry: New and Previous Results Combined. Metrologia, 54, 748-762. http://sci-hub.tw/10.1088/1681-7575/aa7b4a https://doi.org/10.1088/1681-7575/aa7b4a

[21] Newell, D.B., et al. (2017) The CODATA 2017 Values of h, e, k, and NA. Metrologia, 54, 1-6. http://sci-hub.tw/10.1088/1681-7575/aa950a

[22] Mohr, P.J., Newell, D.B., Taylor, B.N. and Tiesinga, E. (2018) Data and Analysis for the CODATA 2017 Special Fundamental Constants Adjustment. Metrologia, 55, 125-146. http://iopscience.iop.org/article/10.1088/1681-7575/aa99bc/pdf https://doi.org/10.1088/1681-7575/aa99bc

[23] Pitre, L., Plimmer, M.D., Sparasci, F. and Himbert, M.E. (2018) Determinations of the Boltzmann Constant. Comptes Rendus Physique, 1-11.

[24] Gaiser, C. and Fellmuth, B. (2012) Low-Temperature Determination of the Boltzmann Constant by Dielectric-Constant Gas Thermometry. Metrologia, 49, 4-7. https://doi.org/10.1088/0026-1394/49/1/L02

[25] Gaiser, C., et al. (2013) Improved Determination of the Boltzmann Constant by Dielectric-Constant Gas Thermometry. Metrologia, 50, 7-11. http://sci-hub.tw/10.1088/0026-1394/50/6/L7 https://doi.org/10.1088/0026-1394/50/6/L7

[26] Christof, C., Thorsten, Z. and Fellmuth, B. (2015) Dielectric-Constant Gas THERMOMETRY. Metrologia, 52, 217-226. http://sci-hub.tw/10.1088/0026-1394/52/5/S217 https://doi.org/10.1088/0026-1394/52/5/S217

[27] Gaiser, C., et al. (2017) Final Determination of the Boltzmann Constant by Dielectric-Constant Gas Thermometry. Metrologia, 54, 280-289.

http://sci-hub.tw/10.1088/1681-7575/aa62e3 https://doi.org/10.1088/1681-7575/aa62e3

[28] Fischer, J., et al. (2018) The Boltzmann Project. Metrologia, 55, 1-36. http://sci-hub.tw/10.1088/1681-7575/aaa790 https://doi.org/10.1088/1681-7575/aaa790

[29] Karshenboim, S.G. (2013) Progress in the Accuracy of the Fundamental Physical Constants: 2010 CODATA Recommended Values. UFN, 183, 935-962. https://goo.gl/rj8RJK

[30] Benz, S.P., et al. (2011) An Electronic Measurement of the Boltzmann Constant. Metrologia, 48, 142-153. https://doi.org/10.1088/0026-1394/48/3/008 
[31] Qu, J., et al. (2015) Improved Electronic Measurement of the Boltzmann Constant by Johnson Noise Thermometry. Metrologia, 52, 242-256. https://doi.org/10.1088/0026-1394/52/5/S242

[32] Truong, G.-W., et al. (2015) Atomic Spectroscopy for Primary Thermometry. Metrologia, 52, 324-342. http://sci-hub.tw/10.1088/0026-1394/52/5/S324 https://doi.org/10.1088/0026-1394/52/5/S324

[33] Qu, J. (2017) An Improved Electronic Determination of the Boltzmann Constant by Johnson Noise Thermometry. Metrologia, 54, 549-558.

http://sci-hub.tw/10.1088/1681-7575/aa781e https://doi.org/10.1088/1681-7575/aa781e

[34] Flowers-Jacobs, N.E. (2017) A Boltzmann Constant Determination Based on Johnson Noise Thermometry. Metrologia, 54, 730-737. https://doi.org/10.1088/1681-7575/aa7b3f

[35] Urano, C., Yamazawa, K. and Kaneko, N.-H. (2017) Measurement of the Boltzmann Constant by Johnson Noise Thermometry Using a Superconducting Integrated Circuit. Metrologia, 54, 847-855. http://sci-hub.tw/10.1088/1681-7575/aa7cdd https://doi.org/10.1088/1681-7575/aa7cdd

[36] Daussy, C. (2007) Direct Determination of the Boltzmann Constant by an Optical Method. Physical Review Letters, 98, 250801. https://doi.org/10.1103/PhysRevLett.98.250801

[37] Casa, G. (2008) Primary Gas Thermometry by Means of Laser-Absorption Spectroscopy: Determination of the Boltzmann Constant. Physical Review Letters, 100, Article ID: 200801. https://doi.org/10.1103/PhysRevLett.100.200801

[38] Djerroud, K., et al. (2009) Measurement of the Boltzmann Constant by the Doppler Broadening Technique at a $3.8 \times 10^{-5}$ Accuracy Level. C. R. Physique, 10, 883-893. https://doi.org/10.1016/j.crhy.2009.10.020

[39] Lemarchand, C., et al. (2011) Progress towards an Accurate Determination of the Boltzmann Constant by Doppler Spectroscopy. New Journal of Physics, 13, Article ID: 073028.

[40] Hashemi, R., Povey, C., Derksen, M., Naseri, H., Garber, J. and Predoi-Cross, A. (2014) Doppler Broadening Thermometry of Acetylene and Accurate Measurement of the Boltzmann Constant. The Journal of Chemical Physics, 141, Article ID 214201. https://doi.org/10.1063/1.4902076

[41] Fasci, E. (2015) The Boltzmann Constant from the $\mathrm{H}_{2}{ }^{18} \mathrm{O}$ vibration-Rotation Spectrum: Complementary Tests and Revised Uncertainty Budget. Metrologia, 52, 233-241. https://doi.org/10.1088/0026-1394/52/5/S233 